\title{
MicroRNA profiling in murine liver after partial hepatectomy
}

\author{
IOANNIS CHAVELES ${ }^{1}$, APOSTOLOS ZARAVINOS ${ }^{2}$, IOANNIS G. HABEOS ${ }^{3}$, DIMITRIOS D. KARAVIAS ${ }^{1}$, \\ IOANNIS MAROULIS ${ }^{1}$, DEMETRIOS A. SPANDIDOS ${ }^{2}$ and DIONISIOS KARAVIAS ${ }^{1}$
}

\author{
${ }^{1}$ Surgical Department, Medical School, University of Patras, 26504 Patras; ${ }^{2}$ Laboratory of Virology, Medical School, \\ University of Crete, 71110 Heraklion, Crete; ${ }^{3}$ Medical Department, Medical School, University of Patras, 26504 Patras, Greece
}

Received January 9, 2012; Accepted February 3, 2012

DOI: 10.3892/ijmm.2012.902

\begin{abstract}
Liver is uniquely capable to repair itself after injury. Multiple molecular and biochemical processes initiated after partial hepatectomy, lead to proliferation of all cells within the liver. MicroRNAs (miRNAs) are a class of highly abundant non-coding RNA molecules that cause post-transcriptional gene repression and are involved in several biological processes including cell cycle regulation and differentiation. In this study, we examined the expression levels of miRNAs in liver tissue received from control mice (L0) and compared them with the corresponding levels in liver tissue $12 \mathrm{~h}$ after liver regeneration induced by $2 / 3$ partial hepatectomy (L12). MiRNA expression was investigated using microRNA profiling. Further qPCR analysis was used for validation of the differentially expressed miRNAs at an early stage of liver regeneration, induced by 2/3 partial hepatectomy. TargetScan and Gene Ontology (GO) analyses were performed in order to identify the possible miRNA target genes and their ontology, respectively. A subset of miRNAs was found to be differentially expressed during liver regeneration. Mmu-miR-21 and mmu-miR-30b* showed the higher levels of up-regulation in liver tissue from the hepatectomized mice at the end of the experiment (L12) compared to the sham operated mice (L0). Mmu-miR-21 up-regulation was further confirmed by qPCR. In situ hybridization (ISH) revealed that mmu-miR-21 exhibited the higher levels of expression at $12 \mathrm{~h}$ post hepatectomy. On the contrary, mmu-miR-34c , mmu-miR-144, mmu-miR-207, mmu-miR-207, mmu-miR-451, mmu-miR-582-3p and mmu-miR-290-5p exhibited $<0.5$ downregulation in liver tissue after partial hepatectomy in L12 vs. L0 mice. The results from microarray and qPCR analyses were in good agreement. In conclusion, our results provide important information regarding the differentially expressed miRNAs in murine liver tissue before and after partial hepatectomy. The
\end{abstract}

Correspondence to: Dr Ioannis G. Habeos, Department of Internal Medicine, Medical School, University of Patras, 26504 Patras, Greece

E-mail: ihabeos@med.upatras.gr

Key words: liver regeneration, mmu-miR-21, microRNA profiling, qPCR, in situ hybridization early up-regulation of mmu-miR-21 during the process of liver regeneration suggests a regulatory role in liver regeneration in vivo.

\section{Introduction}

Partial hepatectomy (PH) represents the most commonly used model for the study of liver regeneration. It is a very complex and well-orchestrated phenomenon that is carried out by the participation of all mature liver cell types. The extent of hepatocyte proliferation is directly proportional to the amount of resected liver tissue and $2 / 3$ partial hepatectomy $(2 / 3 \mathrm{PH})$ leads to a highly synchronized hepatocyte cell-cycle entry and progression. The first phase, known as the 'priming phase', occurs in the first hours after PH and poises the hepatocytes to enter the G1 phase and to become receptive to growth factors. The second phase corresponds to an increased metabolic demand imposed on the remnant liver. During this phase, among other metabolic changes, transient hypoglycemia is suggested to induce systemic lipolysis followed by a lipid droplets accumulation in the hepatocytes. Between 36-42 h after 2/3 PH, most hepatocytes are in the $\mathrm{S}$ phase of the cell cycle. The remnant liver lobes will compensate for lost tissue and recover the initial liver mass in less than 2 weeks. The last and much less studied step is the termination of liver regeneration. A large number of genes are involved in liver regeneration, but the essential circuitry required for the process may be categorized into 3 networks: cytokine, growth factor and metabolic.

MicroRNAs (miRNAs) are short regulatory RNAs of approximately 22 nucleotides in length (1). They bind to the 3'-untranslated region (3'UTR) of target mRNA based on sequence complementarity and result in the degradation or suppression of translation of the target miRNA (2). Nevertheless, new findings reveal a new mode of action of miRNAs by which they may regulate gene expression, by binding not only to regions confined to the $3^{\prime} \mathrm{UTR}$, but to the complete sequence (promoter, 5'UTR, CDS and 3'UTR) of a gene (3-5). It is thought that more than $30 \%$ of human genes are post-transcriptionally regulated by miRNAs $(6,7)$. There has been growing interest in understanding the specific role of miRNAs in regulating cellular processes, such as cell growth, proliferation, apoptosis, stress response and metabolism (8-10).

There are previous studies implicating miRNAs in the regeneration of zebrafish fins (11), regeneration in planarian worms (12) and wound healing (13). Mice with hepatocyte- 
specific inactivation of DGCR8, which is required for miRNA biogenesis, have also been generated. They develop normally after adulthood but their hepatocytes fail to transit into the $S$ phase after PH (7). In a recent study miR-21 was found to be up-regulated during the proliferative phase of liver regeneration. MiR-21 has been shown to target Pellino- 1 and inhibit the NF- $\mathrm{KB}$ signalling (14).

Since recent studies have identified miRNAs as important regulators of gene expression, modulating critical cellular functions of hepatocytes, such as proliferation, apoptosis, lipid metabolism and liver regeneration, we aimed to identify the differentially expressed miRNAs during an early phase of liver regeneration. To this effect, the expression of liver miRNAs was investigated using miRNA profiling and $\mathrm{qPCR}$, in total RNA extracted from murine liver tissue $12 \mathrm{~h}$ after $2 / 3 \mathrm{PH}$.

\section{Materials and methods}

Mice, $P H$ and $R N A$ isolation. Twenty male wild-type mice C57BL6J (8 weeks old) fed a standard diet were used for the liver regeneration experiment. Mice were housed in the animal facility of the University of Patras Medical School in temperature-, light- and humidity-controlled rooms with a 12-h light/dark cycle. All animal procedures were approved by the Institutional Review Board of the University of Patras Medical School and were in accordance with EC Directive 86/609/EEC. A 2/3 PH was performed on 10 mice according to a previously published standardized protocol (15) whereby the median and left lobes of the liver were removed and another 10 sham-operated mice were used as the control group. The liver was allowed to regenerate and mice were sacrificed $12 \mathrm{~h}$ after operation. Liver samples were collected from the sham-operated mice (L0) and the hepatectomized mice at the end of the experiment (L12).

Liver tissue RNA extraction. Liver tissue was excised from mice and was immediately submerged in RNAlater solution (Ambion, Foster City, CA). For miRNA profiling, total RNA was isolated from pooled livers from the L0 and L12 mice, whereas for qPCR experimentation, total RNA was prepared from individual liver samples, using the TRIzol ${ }^{\circledR}$ reagent (Invitrogen, Carlsbad, CA) as the manufacturer instructed. The quality of the total RNA was verified by an Agilent 2100 Bioanalyzer profile (Agilent Technologies, Santa Clara, CA).

miRNA profiling. Total RNA (1 $\mu \mathrm{g})$ from sample and reference were labeled with $\mathrm{Hy} 3^{\mathrm{TM}}$ and $\mathrm{Hy}^{\mathrm{TM}}$ fluorescent label, respectively, using the miRCURY ${ }^{\mathrm{TM}}$ LNA Array power labeling kit (Exiqon, Vedbaek, Denmark). The Hy3-labeled samples and the Hy5-labeled samples were mixed pair-wise and hybridized to the miRCURY LNA array version 10.0 (Exiqon), which contains capture probes targeting all miRNAs for all species registered in the miRBASE version 11.0 at the Sanger Institute. The hybridization was performed according to the miRCURY LNA array manual using a Tecan HS4800 hybridization station (Tecan, Grödig, Austria). After hybridization, the microarray slides were scanned and stored in an ozone-free environment (ozone level below $2.0 \mathrm{ppb}$ ) in order to prevent potential bleaching of the fluorescent dyes. The miRCURY LNA array microarray slides were scanned using the Agilent G2565BA Microarray Scanner System (Agilent Technologies, Inc.) and the image analysis was carried out using the ImaGene 8.0 software (BioDiscovery, Inc., El Segundo, CA).

Microarray data filtering, background correction and normalization. Filtering was performed based on the signal intensity. Spots with no signal above the background (flags 1 and 2) were detected and removed. Background correction was performed to remove non-biological contributions ('background') to the measured signal, by subtracting the median global background from the median local background from the signal intensity. A threshold of 1.5 (L12 vs. L0) was set as the cut-off value. Normalization was performed using the Lowess regression algorithm, in order to remove certain systematic biases from microarray data, such as dye effects or intensity dependence. The positive effect from normalization is illustrated on each slide sheet with an M-A plot before and after normalization (Fig. 1). After normalization the spots appeared symmetrically scattered around the horizontal line $(\mathrm{M}=0)$. Normalized data were extracted, pre-processed and sorted with Microsoft Excel ${ }^{\circledR}$.

Slide quality check using spike-in controls. Spike-in co-efficient of variation $(\mathrm{CV})$ values were calculated between 48 replicates for each of the spike-in controls on each slide. CVs did not exceed 30\%, thus reflecting lack of spatial effects on the array (smear across the slide) or misplacement of the grid for annotating the spots.

Clustering. We clustered the miRNAs between the L0 and L12 murine livers, using hierarchical clustering (HCL) and k-means clustering, as previously reported $(16,17)$.

Gene Expression Omnibus (GEO) accession numbers. Array data were deposited at the GEO (National Center for Biotechnology Information) with accession numbers GSM862374 through GSM862375 (GSE35128).

Reverse transcription $(R T)$ and $q P C R$ validation. The miRNA in the total RNA sample was converted to cDNA by reverse transcription using the miRCURY LNA miRNA PCR system First-strand cDNA synthesis kit and a miRNA-specific primer. Using automatic robot stations the RT and qPCR steps were fully automated for excellent reproducibility. The cDNA was amplified by real-time PCR using SYBR ${ }^{\circledR}$ Green master mix and $\mathrm{LNA}^{\mathrm{TM}}$ miRNA-specific primers on a LightCycler 1.5 (Roche Applied Science) thermal cycler, using a 32-well carousel and 25- $\mu$ l volume capillaries. The following 8 miRNAs were selected for qPCR validation of their expression: mmu-miR-21, mmu-miR-101a, mmu-miR-101b, mmu-miR-669f, mmu-miR-207, mmu-miR-451, mmu-miR-582-3p and mmu-miR-34c. All runs included no template and RT-minus controls. A 5-fold serial dilution of pooled cDNA samples was generated for each assay, in order to calculate the qPCR efficiency. All reactions were performed in triplicate. Before proceeding with data normalization, a technical quality assessment was performed based on the results of the melting curve, serial dilution curve and no-template-controls. The stability and ranking of the endogenous controls was also calculated with the SLqPCR algorithim. For every endogenous control gene, the pair-wise variation with all other endogenous controls was determined as a gene stability measurement M. An M-value below 1.5 was recommended and genes with expres- 

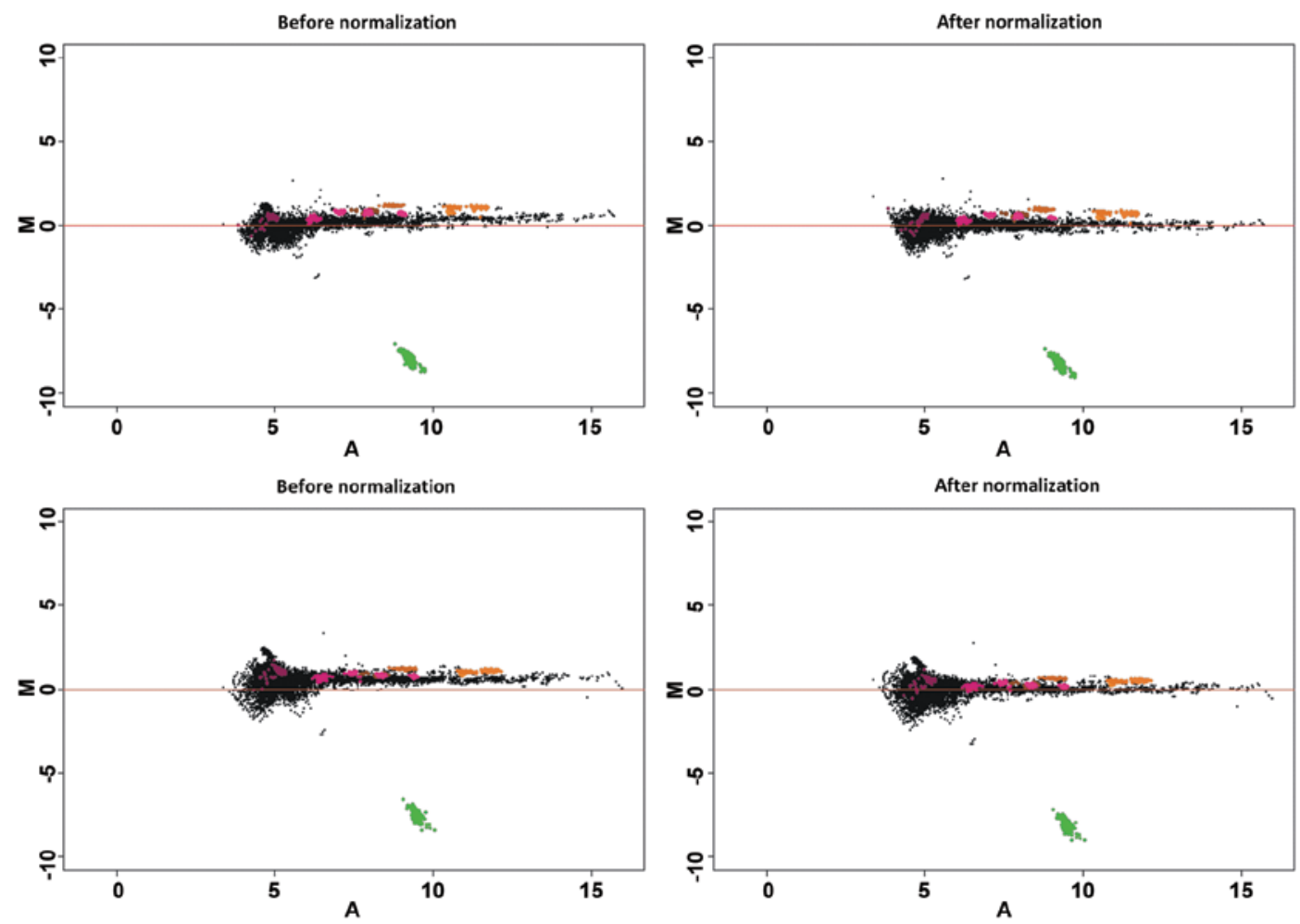

Figure 1. The positive effect from normalization is illustrated on each slide with an M-A plot before and after normalization. After normalization the spots appear symmetrically scattered around the horizontal line $\mathrm{M}=0$. After normalization, the difference between the two channels ( $\mathrm{M}$ ) is independent of the average intensity level of the two channels. The green spots are Hy3 controls spotted directly on the array surface. The orange/pink spots are spike-in controls.

sion stability above 1.5 were considered unstable across the samples and unsuitable for endogenous controls in this experiment. Mmu-miR-103 and mmu-miR-let-7b were considered acceptable and used for normalization, whereas mmu-miR-191 was excluded due to too high $\mathrm{Cp}$-values for one of the negative controls (18) (Fig. 2). Relative expression was performed as described previously $(19,20)$ using the $\Delta \Delta \mathrm{Ct}$ method, as follows (data were standardized by $\log _{2}$ transformation):

$$
\text { Relative expression }=\frac{\left.E_{\text {miRNA }} \Delta C \text { (sampleA-sample } B\right)}{N F}
$$

In situ hybridization (ISH). The in situ hybridization was carried out as previously described with minor modifications (21). Briefly, 4- $\mu \mathrm{m}$ sections of paraffin-embedded, formalin-fixed liver tissue samples were prepared. Slices were digested with pepsin for $30 \mathrm{~min}$ at room temperature. Digoxigenin-labeled locked nucleic acid probes $(2 \mathrm{pmol} / \mu \mathrm{l})$ (Exiqon, Woburn, MA) were used for the hybridization $\left(37^{\circ} \mathrm{C}\right.$ for $12 \mathrm{~h}$ ) and their presence visualized via anti-digoxigenin with antidigoxigenin-alkaline phosphatase conjugate $(1: 150$ dilution) for $30 \mathrm{~min}$ at $37^{\circ} \mathrm{C}$. Detection was done with NBT/ BCIP chromogen at $37^{\circ} \mathrm{C}$.

TargetScan, Gene Ontology (GO) and enrichment analyses. A miRNA binds to the 3'UTR of a target mRNA via the 'seed' region of the mature miRNA (nt 2-7). For the 8 most differentially expressed miRNAs, we used TargetScan (http://www.targetscan. org/, release 5.0) analysis in order to identify the possible miRNA target genes. Next, we used GO analysis to obtain an overview of the main classes of biological functions of the genes predicted to be targets for those miRNAs. GO analysis was performed, using the WebGestalt web-tool (http://bioinfo.vanderbilt.edu/ webgestalt) (22). All gene definitions and functions were based on the National Institute of Health databases (http://www. ncbi.nlm.nih.gov/sites/entrez). GO enrichment analysis for the putative targets of miRNAs was also investigated, using the WebGestalt web-tool (http://bioinfo.vanderbilt.edu/webgestalt) (22). The hypergeometric test, with Bonferroni correction was used for enrichment evaluation analysis. The $\mathrm{R}$ function adjP was used in order to adjust the nominal p-values of the large number of categories at the same time. The significance level for the adjusted p-value was set at 0.01 and the number of minimum genes for a category was set at 2 .

Statistical analysis. Normality of the data distribution was checked by Kolmogorov-Smirnov test. Differences in the expression levels in the liver between LO and L12 mice were explored using the t-test. Numerical values are expressed as the means \pm standard deviation (SD). Statistical significance was set at the $95 \%$ level $(\mathrm{p}<0.05)$.

\section{Results}

In the present study, we examined the expression levels of 598 mmu-miRNAs in liver tissue received from control mice (L0) and compared them with the corresponding expression in liver 


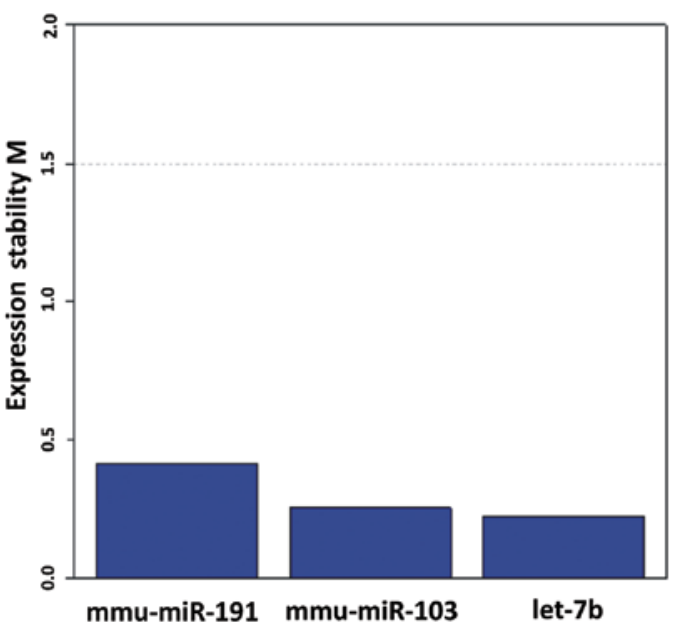

Figure 2. Stability of endogenous controls. For every endogenous control gene the pair-wise variation with all other endogenous controls is determined as a gene stability measurement $\mathrm{M}$ calculated with the SLqPCR algorithim. An expression stability M-value below 1.5 is recommended and a gene with expression stability above 1.5 was considered unstable across the samples and unsuitable for endogenous controls in this experiment.
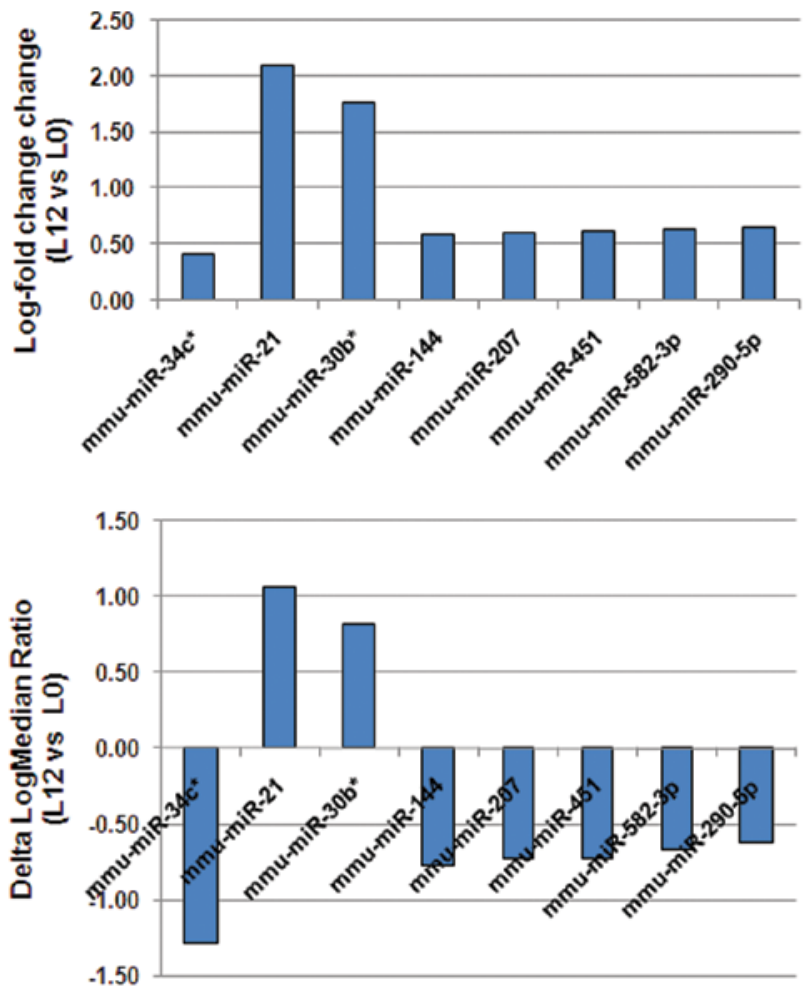

Figure 3. The upper half part of the diagram shows the Log-fold change (L12 vs. L0) of the microRNAs that passed the filtering criteria on variation across samples. For better visualization of the expression results, the lower half part depicts the Delta Log Median Ratio $> \pm 0.58$ equal to (Fold change) $>1.5$ of same microRNAs. Each bar represents the Log Median Ratio between the L12 and LO samples. A positive Delta Log Median Ratio indicates up-regulation in the L12 sample and a negative Delta Log Median Ratio indicates down-regulation in the L12 sample compared to the L0 sample.

tissue $12 \mathrm{~h}$ after liver regeneration induced by $2 / 3$ partial hepatectomy (L12).

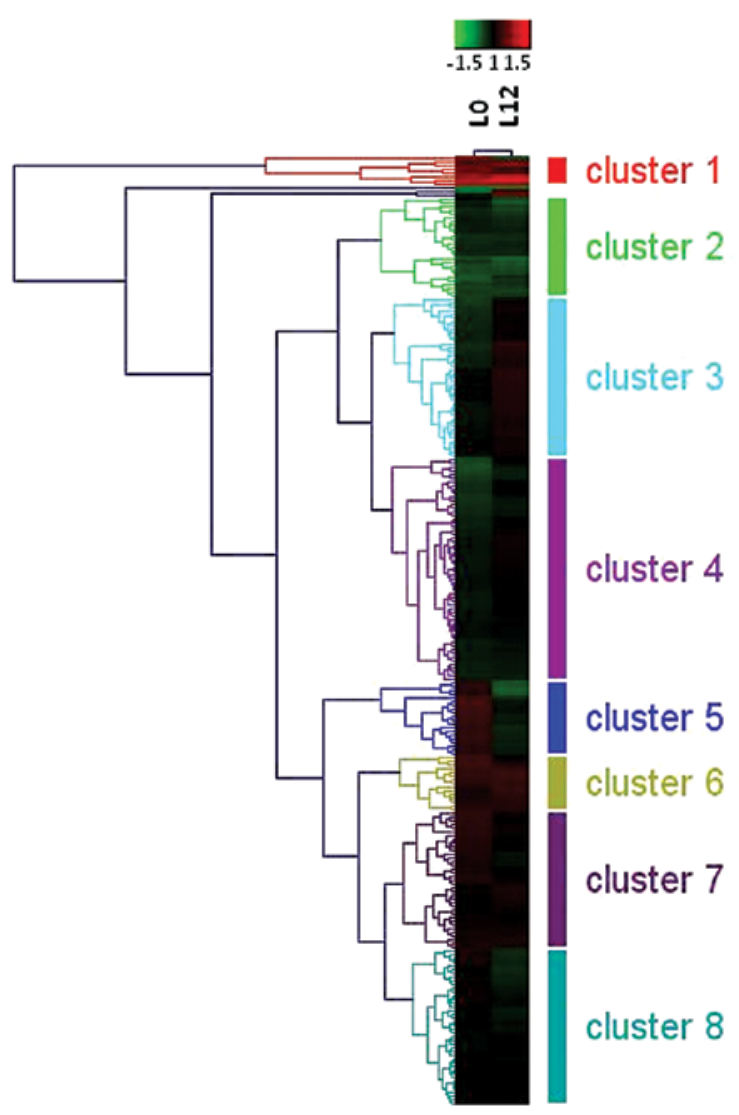

Figure 4. Unsupervised hierarchical cluster analysis diagram based on 598 probe sets with highest variation in L12 vs. L0 mice. Color saturation is directly proportional to measured expression ratio magnitude. Rows represent individual probe set. Columns represent the experimental samples L0 and L12. Red bars indicate high expression. Green bars indicate low expression.

Microarray profiling. Eight out of 598 miRNAs passed the filtering criteria on variation across samples; Delta Log Median Ratio $(\Delta \mathrm{LMR})> \pm 0.58$ equal to [Fold change] $>1.5$. The 8 most differentially expressed (DE) miRNAs in the liver tissue in the L12 mice vs. the L0 mice were: mmu-miR-34c ${ }^{*}$, mmu-miR-21, mmu-miR-30b* , mmu-miR-144, mmu-miR-207, mmu-miR-451, mmu-miR-582-3p and mmu-miR-290-5p. The difference in $\triangle \mathrm{LMR}$ between L12 and L0 mice was calculated. Moreover, the average Log Median Ratio was converted to fold change as follows: Fold change $=2^{\Delta \mathrm{LMR}}$ (Fig. 3). Mmu-miR-21 and mmu-miR-30b ${ }^{*}$ were overexpressed in the L12 mice vs. the L0 mice, whereas, the other 6 miRs (mmu-miR-34c* ${ }^{*}$ mmu-miR-144, mmu-miR-207, mmu-miR-451, mmu-miR-582-3p and mmu-miR-290-5p) exhibited lower expression levels in the L12 mice vs. the $\mathrm{L} 0$ ones.

Hierarchical clustering. We performed average linkage hierarchical clustering using Euclidian distance. A detailed tree and cluster image, depicting the names of each mmu-miRNA is depicted in Fig. 4. Eight gene clusters were noted. Regarding the DE miRs, mmu-miR-34c* belonged in the first cluster, showing significantly higher expression in the L0 vs. the L12 mice. On the contrary, mmu-miR-21 and mmu-miR-30b* were significantly up-regulated in the L12 vs. the L0mice. Our interest was also focused on clusters 5 (mmu-miR-144, mmu-miR-207, 


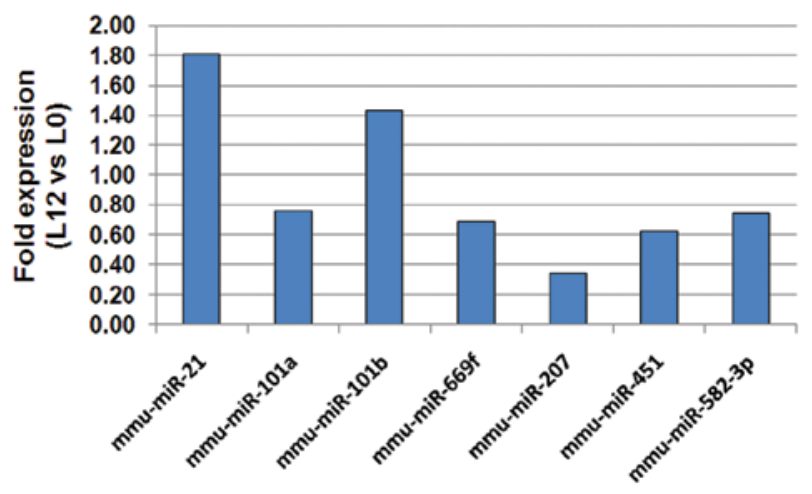

Figure 5. Fold expression in the 8 most differentially expressed mmu-miRs in the $\mathrm{L} 12$ vs. the $\mathrm{L} 0$ mice, as acquired by qPCR.

mmu-miR-451, mmu-miR-582-3p and mmu-miR-290-5p) and 8 , where the corresponding mmu-miRNAs were either down-regulated in the L0 mice vs. the L12 mice (cluster 3), or up-regulated in the L0 mice vs. the L12 mice (clusters 5 and 8), respectively.

$q P C R$ validation of expression. From the 8 most differentially expressed mmu-miRs (L12 vs. L0 in microarray data), 5 miRs (mmu-miR-21,mmu-miR-207,mmu-miR-451,mmu-miR-582-3p, mmu-miR-34c) were also tested by qPCR. Three more miRs (mmu-miR-101a, mmu-miR-101b and mmu-miR-669f) that were marginally differentially expressed in the microarrays [both for mmu-miR-101a and mmu-miR-101b, $\Delta$ LMR $=0.50$ (fold change L12 vs. L0 =1.41); and for mmu-miR-669f, $\Delta$ LMR $=-0.49$ (fold change L12 vs L0, 0.71)] were also included in the qPCR experiment. After normalizing the expression levels with the corresponding geometric mean value of the reference genes (mmu-miR-191, mmu-miR-103 and mmu-miR-let-7b), the samples were checked for outliers to be excluded. The qPCR verified the expression profile of the differentially expressed miRs, as acquired by the microarrays. The fold changes were calculated using the $\Delta \Delta \mathrm{Ct}$ comparative quantification method. Data were corrected for assay-specific PCR efficiency (miRNA

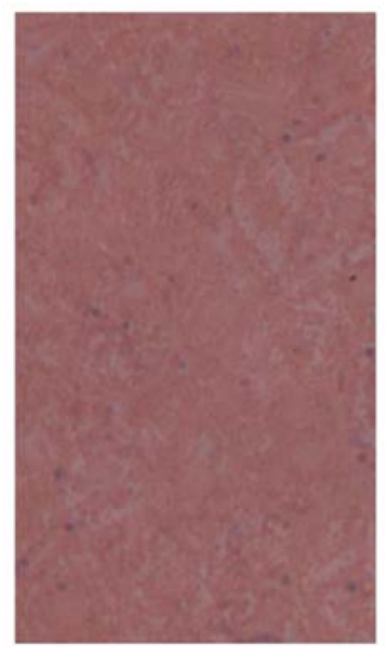

miR-21: Negative control

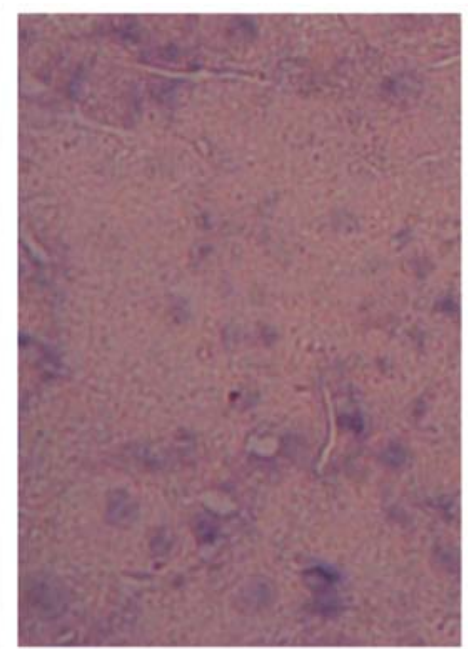

miR-21: Before hepatectomy

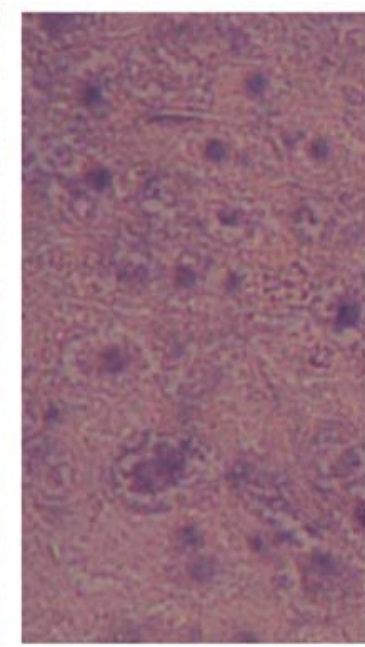

miR-21: 1 hr post-PHx

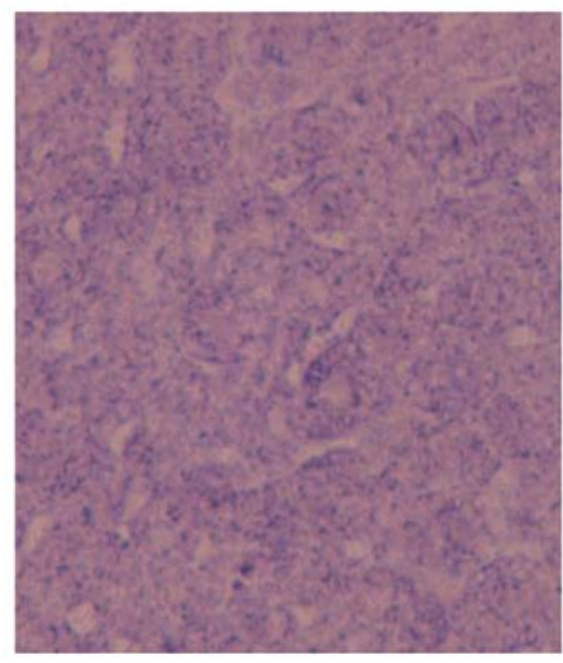

miR-21: $6 \mathrm{hrs}$ post-PHx

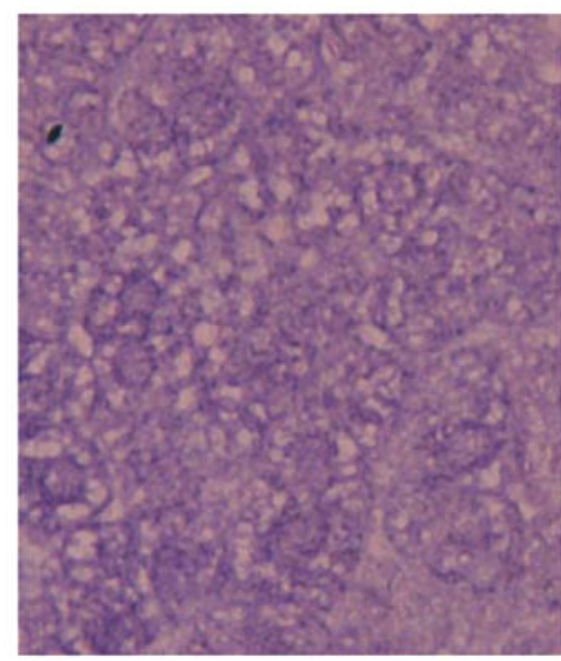

miR-21: $12 \mathrm{hrs}$ post-PHx

Figure 6. In situ hybridization (ISH) of mmu-miR-21 before hepatectomy, 1, 6 and $12 \mathrm{~h}$ post hepatectomy, respectively. Mmu-miR-21 exhibited the highest expression levels at $12 \mathrm{~h}$ post hepatectomy. 
or endogenous control) and normalized with a normalization factor based on three endogenous controls: mmu-miR-191, mmu-miR-103 and mmu-miR-let-7b. The relative fold expression of the aforementioned miRs in L12 vs. L0 mice is indicated in Fig. 5. Our qPCR analysis distinguished that mmu-miR-21 is the most significantly overexpressed miRNA in the L12 mice vs. the L0 ones (1.808-fold change; $\mathrm{p}<0.01$; $\mathrm{t}$-test), among the miRs studied. The increased levels of mmu-miR-21 were further validated by ISH. Specifically, increased mmu-miR-21 levels were detected post-hepatectomy compared to pre-hepatectomy and its highest levels were noticed at $12 \mathrm{~h}$ post-hepatectomy (Fig. 6).

Correlation between microrrays and $q P C R$. The results received from the microarray and $\mathrm{qPCR}$ analyses were in good agreement. The relative expressions were based on four technical replicates. Pearson's correlation ( $r$ ) between microarrays and $\mathrm{qPCR}$ was $0.881(\mathrm{p}=0.119)$ (Fig. 7). qPCR analysis for mmu-miR-34c*, mmu-miR-30b*, mmu-miR-144 and mmumiR-290-5p revealed extremely low expression levels, therefore the $\triangle \mathrm{LMR}$ data are shown as not available (N/A). Apart from the case of mmu-miR-30b*, this underexpression is in good agreement with the data obtained from our microarray experimentation.

TargetScan and GO enrichment analysis. The GO is divided into 3 ontologies that were found to yield information common to all living organisms. The Ontology of Cellular Component (CC) describes the places in the cell where gene products would be found. The Ontology of Molecular Function (MF) describes the biochemical activity of a gene product without specifying the time or the space where this event occurred. It is a usual feature for a molecular function term to be directly characterized by its annotated gene product. The Ontology of Biological Process (BP) consists of one or more ordered assemblies of functions. There is a considerable relevance between molecular function and biological process ontologies.

No results could be received for mmu-miR-34c* and mmumiR-30b* through TargetScan analysis. Seventy-eight putative gene targets were revealed for mmu-miR-21. These participate significantly in various BPs, namely: positive regulation of cellular process, positive regulation of biological process, regulation of transcription from RNA polymerase II promoter, cellular macromolecule metabolic process, transcription from RNA polymerase II promoter, regulation of metabolic process, regulation of cellular metabolic process, regulation of macromolecule metabolic process, positive regulation of macromolecule biosynthetic process and positive regulation of cellular biosynthetic process $(\operatorname{adj} \mathrm{P}<0.001)$. The ontology of the significant MFs identified functions such as protein binding, binding and transcription factor activity (adjP<0.001) (Table I). TargetScan analysis for mmu-miR-144 revealed 439 putative gene targets. These targets were shown to participate significantly in BPs, such as anatomical structure development, regulation of RNA metabolic process, system development, regulation of primary metabolic process, regulation of gene expression, regulation of metabolic process, multicellular organismal development, DNA-dependent regulation of transcription, organ development and regulation of macromolecule metabolic process $(\operatorname{adj} \mathrm{P}<0.001)$. The MF and $\mathrm{CC}$ of the majority of the genes was in the nucleus. Analysis for mmu-miR-207 revealed 100 puta-

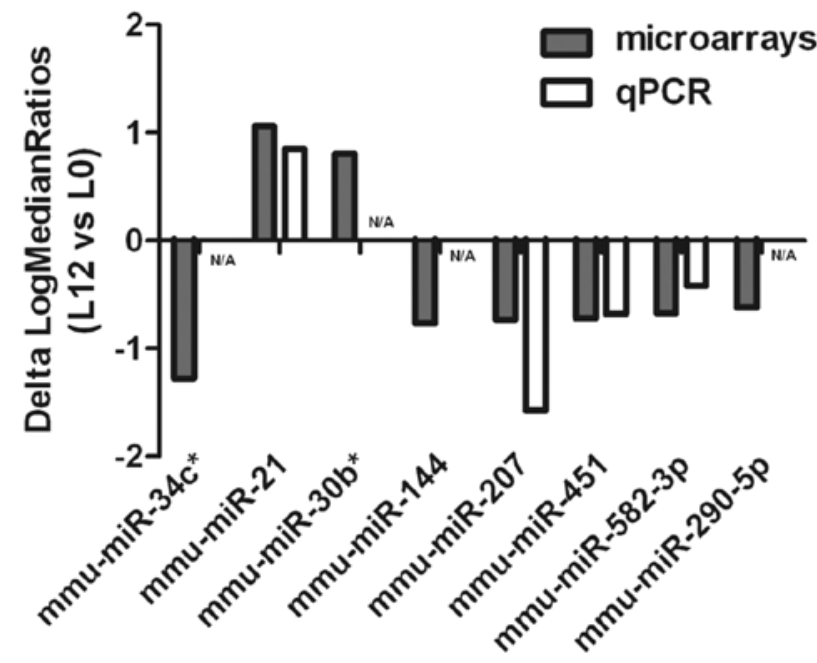

Figure 7. Correlation between microrrays and qPCR experimentation. The fold changes were calculated using the $\Delta \Delta \mathrm{Ct}$ comparative quantification method. Mmu-miR-34c was not detected in the $\mathrm{qPCR}$, hence no fold change was calculated. We identified a good agreement between the microarrays and the qPCR results [Pearson's correlation $(r)=0.881$ ].

tive gene targets, which were shown to participate in transferase activity molecular functions, such as transferring glycosyl groups, transferring hexosyl groups, UDP-glycosyltransferase activity and glucuronosyltransferase activity $(\operatorname{adj} \mathrm{P}<0.001)$. Analysis for mmu-miR-451 revealed 6 putative gene targets. However, no statistically significant results were obtained from the GO enrichment. Mmu-miR-582-3p was shown to have 71 putative gene targets, which participate significantly in various BPs, such as regulation of gene expression, regulation of cellular biosynthetic process, regulation of macromolecule biosynthetic process. Similarly, various CC ontologies were identified, such as nucleus genes and cortical cytoskeleton. Mmu-miR-290-5p was shown to have 122 putative gene targets, participating significantly in BP ontologies such as organ morphogenesis, etc., in MF ontologies, such as transferase activities and CC ontologies, such as intracellular membrane-bounded organelle or nucleus.

\section{Discussion}

In the present study, the differential expression levels of 598 miRNAs in the regenerated mouse liver $12 \mathrm{~h}$ after $\mathrm{PH}$, compared to the baseline levels (L0), was investigated by microarray profiling and further confirmed by qPCR. The most important finding of our study was the induction of mmu-miR-21 posthepatectomy, with higher levels at $12 \mathrm{~h} \mathrm{PH}$. Mmu-miR-21 is a known promoter of proliferation in cancer (23). Previous studies have shown that mmu-miR-21 is transcriptionally regulated by activation protein 1 (AP-1) (24) and signal transducer and activator of transcription 3 (STAT3) (25), two proteins that are activated early in liver regeneration. It is also known that miRNAs control hepatocyte proliferation during liver regeneration (26). Our findings are in agreement with a previous study showing that mmu-miR-21 is up-regulated during the proliferative phase of mouse liver regeneration, it targets Pellino-1, and 
Table I. Putative mmu-miR-21 target genes along with each gene's ontology.

mmu-miR-21 targets $(\operatorname{adj} P<0.05)$

Biological process $\quad$ Molecular function

Positive regulation of cellular process $(\operatorname{adjP}=9.33 \mathrm{e}-06)$

I112a, Sox5, Ppara, Peli1, Yap1, Gata2, Nfat5,

Thrb, Glis2, Alx1, Acvr1c, Nfib, Sox2, Jag1,

Rbpj, Map3k1, Ntf3, Notch2, Il21, Tgfbr2

Positive regulation of biological process $(\operatorname{adj} P=1.39 \mathrm{e}-05)$

Il12a, Cd247, Sox5, Ppara, Peli1, Yap1, Gata2,

Nfat5, Thrb, Glis2, Alx1, Acvr1c, Nfib, Sox2,

Jag1, Rbpj, Map3k1, Ntf3, Notch2, I121, Tgfbr2

Regulation of transcription from RNA polymerase II promoter $(\operatorname{adj} P=4.45 e-05)$

Cbx4, Alx1, Sox7, Nfib, Sox2, Rbpj, Sox5, Ntf3, Ppara, Yap1, Gata2, Nfat5, Thrb, Glis2

Cellular macromolecule metabolic process $(\operatorname{adjP}=7.75 e-05)$ Prpf4b, Klf6, Sox5, Dnajc16, Peli1, Yap1, Gata2, Mrp19, Nfat5, Thrb, Asf1a, Alx1, Sox7, Acvr1c, Map2k3, Mapk10, Ppp1r3b, Nfib, Sox2, Map3k1, Rbpj, Tnks, Npas3, Il21,Btg2, Tgfbr2, Adnp, Fbxo11, Cbx4, Crebl2, Ppara, Glis2, Mrp149, Stk40, Ube2d3, Dnaja2, Ntf3, Notch2, Wwp1, Cdc25a

Transcription from RNA polymerase II promoter (adjP=8.83e-05)

Cbx4, Alx1, Sox7, Nfib, Sox2, Rbpj, Sox5, Ntf3, Ppara, Yap1, Gata2, Nfat5, Thrb, Glis2

Regulation of metabolic process $(\operatorname{adjP}=9.84 \mathrm{e}-05)$ Cbx4, Klf6, Spry2, Sox5, Creb12, Ppara, Peli1, Yap1, Gata2, Nfat5, Asf1a, Thrb, Glis2, Alx1, Sox7, Map2k3, Nfib, Ube2d3, Sox2, Rbpj, Map3k1, Ntf3, Notch2, Npas3, Il21, Btg2,

Tgfbr2, Adnp

Regulation of cellular metabolic process $(\operatorname{adjP}=\mathbf{0 . 0 0 0 1})$ Cbx4, Klf6, Spry2, Sox5, Crebl2, Ppara, Peli1, Yap1, Gata2, Nfat5, Asf1a, Thrb, Glis2, Alx1, Sox7, Map2k3, Nfib, Sox2, Rbpj, Map3k1, Ntf3, Notch2, Npas3, Il21, Btg2, Tgfbr2, Adnp

Regulation of macromolecule metabolic process $(\operatorname{adj} P=0.0002)$

Cbx4, Klf6, Sox5, Crebl2, Ppara, Peli1, Yap1, Gata2, Nfat5, Asf1a, Thrb, Glis2, Alx1, Sox7, Map2k3, Nfib, Ube2d3, Sox2, Rbpj, Map3k1, Ntf3, Notch2, Npas3, Il21, Btg2, Adnp

Positive regulation of macromolecule biosynthetic proces $(\operatorname{adj} \mathrm{P}=\mathbf{0 . 0 0 0 2})$

Alx1, Nfib, Sox2, Sox5, Ntf3, Ppara, Il21, Yap1,

Positive regulation of cellular biosynthetic process $(\operatorname{adjP}=\mathbf{0 . 0 0 0 3})$

Alx1, Nfib, Sox2, Sox5, Ntf3, Ppara, I121, Yap1, Gata2, Nfat5, Thrb, Glis2
Protein binding $(\operatorname{adj} \mathbf{P}=\mathbf{1 . 2 4 e - 0 5})$

Pdzd2, Tgfbi, Arhgef12, Dnajc16, Sox5, Peli1, Yap1, Gata2, Nfat5, Asf1a, Thrb, Alx1, Acvr1c, Cxc110, Mapk10, Reck, Sox2, Pdcd4, Rbpj, Map3k1, Btg2, Il21, Tgfbr2, Adnp, Klhdc5, Mprip, Cbx4, Il12a, Cd247, Kcna1, Glcci1, Spry2, Creb12, Rasa1, Ppara, Olr1, Ccl1, Jag1, Dnaja2, Ntf3, Notch2, Wwp1, Pag1

\section{Binding (adjP=0.0002)}

Pdzd2, Prpf4b, Rbms3, Tgfbi, Klf6, Arhgef12, Dnajc16, Sox5, Peli1, Yap1, Gata2, Nfat5, Asf1a, Thrb, Alx1, Sox7, Acvr1c, Cxcl10, Map2k3, Mapk10, Nfib, Reck, Sox2, Pdcd4, Rbpj, Map3k1, Npas3, Btg2, Il21, Tgfbr2, Adnp, Klhdc5, Mprip, Fbxo11, Cbx4, Il12a, Cd247, Kcna1, Glcci1, Spry2, Creb12, Rasa1, Cpeb3, Ppara, Lemd3, Olr1, Rasa2, Glis2, Ccl1, Zadh2, Stk40, Ube2d3, Jag1, Dnaja2, Ntf3, Notch2, Wwp1, Rspo2, Pag1, Rasgrp1

Transcription factor activity $(\mathbf{a d j P}=0.0004)$

Alx1, Sox7, Nfib, Sox2, Sox5, Rbpj, Crebl2, Ppara, Gata2, Adnp, Nfat5, Thrb, Glis2

Transcription regulator activity $(\mathbf{a d j P}=\mathbf{0 . 0 0 2 4})$

Alx1, Sox7, Nfib, Sox2, Sox5, Rbpj, Crebl2, Ppara, Npas3, Yap1, Gata2, Adnp, Nfat5, Thrb, Glis2

Receptor signaling protein serine/threonine kinase activity (adjP=0.0051)

Acvr1c, Mapk10, Tgfbr2, Map3k1

MAP kinase kinase activity $(\mathbf{a d j P}=\mathbf{0 . 0 1 4 8})$

Map2k3, Map3k1

Cytokine receptor binding $(\operatorname{adj} P=0.0222)$

Il12a, Il21, Cxcl10, Ntf3, Ccl1

Protein serine/threonine/tyrosine kinase activity $(\mathbf{a d j} \mathbf{P}=\mathbf{0 . 0 3 7 0})$

Map2k3, Map3k1 
inhibits the NF- $\mathrm{BB}$ signalling (14). Targeting of Pelil by miR-21 could potentially provide the basis for a negative feedback cycle regulating $\mathrm{NF}-\kappa \mathrm{B}$ signalling, since it has been previously reported that NF- $\kappa \mathrm{B}$ up-regulates the miR-21 precursor transcript. Another study of rat liver regeneration after $\mathrm{PH}$, identified among other miRNAs, the induction of miR-21 as early as $3 \mathrm{~h}$ post hepatectomy (27).

It should be noted that mmu-miR-21 is unlikely to be the only differentially expressed miRNA in murine regenerative liver $12 \mathrm{~h}$ after $\mathrm{PH}$, compared to the baseline levels. It is very interesting that the mmu-miR-34 family has potent antiproliferative activities with miR-34c identified as target of p53 that controls cell proliferation $(10,28)$. Since mmu-miR-34c ${ }^{*}$ was found to be down-regulated at $12 \mathrm{~h}$ following $\mathrm{PH}$, its mature strand sequence, mmu-miR-34c, was tested by qPCR analysis, but the expression levels were too low to make any firm conclusions.

Our finding of mmu-miR-207 repression is in agreement with a recent study that showed that $\mathrm{miR}-207$ is down-regulated during rat liver regeneration (29).

Mmu-miR-144 is known to promote erythropoiesis. However, in a recent study miR-144 was shown to impair insulin signalling by inhibiting the expression of insulin receptor substrate 1 levels in a rat model of type 2 diabetes (30). It is conceivable that the down-regulated mmu-miR-144 facilitates insulin/IFG1 signalling and hence liver regeneration.

Mmu-miR-451 seems to have tumor suppressor effects. It is a conditional switch controlling glioma cell proliferation and migration (31) and it inhibits tumor growth of glioblastoma stem cells (32). Its down-regulation during liver regeneration is consistent with this function. Although mmu-miR-451 has never been reported to be involved in liver regeneration before, it has been found to be expressed in rat liver and it is down-regulated in diet induced non-alcoholic fatty liver disease (33). The repression of the tumor suppressor, miR-451, has been shown to be essential for Notch-1-induced oncogenesis in T-ALL (34). It is worth mentioning that Notch-1 signalling is important for liver regeneration (35).

To the best of our knowledge, this is the first report to link mmu-miR-290-5p down-regulation with murine liver regeneration. Its down-regulation by thyrotropin is required for thyroid cell growth (36).

A large number of putative gene targets was identified for each one of the differentially expressed miRNAs between the L12 and L0 mice, using the TargetScan algorithm. Further GO analysis revealed the biological properties and molecular functions of this large number of genes.

In conclusion, in the present study we identified that mmu-miR-21 is induced early during the process of liver regeneration. This result indicates that mmu-miR-21 might be significantly involved in this process. Similarly, mmu-miR$30 b^{*}$ showed increased levels in the L12 vs. the L0 murine livers. However, mmu-miR-21 and mmu-miR-30b* are unlikely to be the only miRNAs involved in liver regeneration. The down-regulation of mmu-miR-34c* ${ }^{*}$ mmu-miR-144, mmu-miR207, mmu-miR-207, mmu-miR-451, mmu-miR-582-3p and mmu-miR-290-5p, is consistent with their known role in cell proliferation generally. However, further investigation is still required to unveil the role that miRNAs play in liver regeneration.

\section{References}

1. Bartel DP: MicroRNAs: genomics, biogenesis, mechanism, and function. Cell 116: 281-297, 2004.

2. Rupaimoole R, Han HD, Lopez-Berestein G and Sood AK: MicroRNA therapeutics: principles, expectations, and challenges. Chin J Cancer 30: 368-370, 2011.

3. Tay Y,Zhang J, Thomson AM, Lim B and Rigoutsos I: MicroRNAs to Nanog, Oct 4 and Sox 2 coding regions modulate embryonic stem cell differentiation. Nature 455: 1124-1128, 2008.

4. Guang S, Bochner AF, Pavelec DM, et al: An Argonaute transports siRNAs from the cytoplasm to the nucleus. Science 321: 537-541, 2008.

5. Place RF,Li LC, Pookot D, Noonan EJ and Dahiya R: MicroRNA373 induces expression of genes with complementary promoter sequences. Proc Natl Acad Sci USA 105: 1608-1613, 2008.

6. Lewis BP, Burge CB and Bartel DP: Conserved seed pairing, often flanked by adenosines, indicates that thousands of human genes are microRNA targets. Cell 120: 15-20, 2005.

7. Ke XS, Liu CM, Liu DP and Liang CC: MicroRNAs: key participants in gene regulatory networks. Curr Opin Chem Biol 7: 516-523, 2003.

8. Le Sage C, Nagel R, Egan DA, et al: Regulation of the p27(Kip1) tumor suppressor by miR-221 and miR-222 promotes cancer cell proliferation. EMBO J 26: 3699-3708, 2007.

9. Poy MN, Eliasson L, Krutzfeldt J, et al: A pancreatic islet-specific microRNA regulates insulin secretion. Nature 432: 226-230, 2004.

10. Corney DC, Flesken-Nikitin A, Godwin AK, Wang W and Nikitin AY: MicroRNA-34b and MicroRNA-34c are targets of p53 and cooperate in control of cell proliferation and adhesionindependent growth. Cancer Res 67: 8433-8438, 2007.

11. Thatcher EJ, Paydar I, Anderson KK and Patton JG: Regulation of zebrafish fin regeneration by microRNAs. Proc Natl Acad Sci USA 105: 18384-18389, 2008.

12. Palakodeti D, Smielewska M and Graveley BR: MicroRNAs from the Planarian Schmidtea mediterranea: a model system for stem cell biology. RNA 12: 1640-1649, 2006.

13. Bostjancic E and Glavac D: Importance of microRNAs in skin morphogenesis and diseases. Acta Dermatovenerol Alp Panonica Adriat 17: 95-102, 2008.

14. Marquez RT, Wendlandt E, Galle CS, Keck K and McCaffrey AP: MicroRNA-21 is upregulated during the proliferative phase of liver regeneration, targets Pellino-1, and inhibits NF-kappaB signaling. Am J Physiol Gastrointest Liver Physiol 298: G535-G541, 2010.

15. Mitchell $\mathrm{C}$ and Willenbring $\mathrm{H}$ : A reproducible and well-tolerated method for $2 / 3$ partial hepatectomy in mice. Nat Protoc 3: 1167-1170, 2008.

16. Zaravinos A, Lambrou GI, Boulalas I, Delakas D and Spandidos DA: Identification of common differentially expressed genes in urinary bladder cancer. PLoS One 6: e18135, 2011.

17. Zaravinos A, Lambrou GI, Volanis D, Delakas D and Spandidos DA: Spotlight on differentially expressed genes in urinary bladder cancer. PLoS One 6: e18255, 2011.

18. Vandesompele J, De Preter K, Pattyn F, et al: Accurate normalization of real-time quantitative RT-PCR data by geometric averaging of multiple internal control genes. Genome Biol 3: RESEARCH0034, 2002.

19. Livak KJ and Schmittgen TD: Analysis of relative gene expression data using real-time quantitative PCR and the $2(-\Delta \Delta \mathrm{C}(\mathrm{T}))$ Method. Methods 25: 402-408, 2001.

20. Radojicic J, Zaravinos A, Vrekoussis T, Kafousi M, Spandidos DA and Stathopoulos EN: MicroRNA expression analysis in triplenegative (ER, PR and Her2/neu) breast cancer. Cell Cycle 10: 507-517, 2011 .

21. Nuovo GJ, Elton TS, Nana-Sinkam P, Volinia S, Croce CM and Schmittgen TD: A methodology for the combined in situ analyses of the precursor and mature forms of microRNAs and correlation with their putative targets. Nat Protoc 4: 107-115, 2009.

22. Zhang B, Kirov S and Snoddy J: WebGestalt: an integrated system for exploring gene sets in various biological contexts. Nucleic Acids Res 33: W741-W748, 2005.

23. Selcuklu SD, Donoghue MT and Spillane C: miR-21 as a key regulator of oncogenic processes. Biochem Soc Trans 37: 918-925, 2009.

24. Fujita S, Ito T, Mizutani T, et al: miR-21 gene expression triggered by AP- 1 is sustained through a double-negative feedback mechanism. J Mol Biol 378: 492-504, 2008. 
25. Loffler D, Brocke-Heidrich K, Pfeifer G, et al: Interleukin-6 dependent survival of multiple myeloma cells involves the Stat3mediated induction of microRNA-21 through a highly conserved enhancer. Blood 110: 1330-1333, 2007.

26. Song G, Sharma AD, Roll GR, et al: MicroRNAs control hepatocyte proliferation during liver regeneration. Hepatology 51: 1735-1743, 2010.

27. Castro RE, Ferreira DM, Zhang X, et al: Identification of microRNAs during rat liver regeneration after partial hepatectomy and modulation by ursodeoxycholic acid. Am J Physiol Gastrointest Liver Physiol 299: G887-G897, 2010.

28. He L, He X, Lim LP, et al: A microRNA component of the p53 tumour suppressor network. Nature 447: 1130-1134, 2007.

29. Raschzok N, Werner W, Sallmon H, et al: Temporal expression profiles indicate a primary function for microRNA during the peak of DNA replication after rat partial hepatectomy. Am J Physiol Regul Integr Comp Physiol 300: R1363-R1372, 2011

30. Karolina DS, Armugam A, Tavintharan S, et al: MicroRNA 144 impairs insulin signaling by inhibiting the expression of insulin receptor substrate 1 in type 2 diabetes mellitus. PLoS One 6: e22839, 2011.
31. Godlewski J, Bronisz A, Nowicki MO, Chiocca EA and Lawler S: microRNA-451: a conditional switch controlling glioma cell proliferation and migration. Cell Cycle 9: 2742-2748, 2010.

32. Gal H, Pandi G, Kanner AA, et al: MIR- 451 and imatinib mesylate inhibit tumor growth of glioblastoma stem cells. Biochem Biophys Res Commun 376: 86-90, 2008.

33. Alisi A, Da Sacco L, Bruscalupi G, et al: Mirnome analysis reveals novel molecular determinants in the pathogenesis of dietinduced nonalcoholic fatty liver disease. Lab Invest 91: 283-293, 2011.

34. Li X, Sanda T, Look AT, Novina CD and von Boehmer H: Repression of tumor suppressor miR-451 is essential for NOTCH1-induced oncogenesis in T-ALL. J Exp Med 208: 663-675, 2011.

35. Wakabayashi N, Shin S, Slocum SL, et al: Regulation of notch1 signaling by nrf2: implications for tissue regeneration. Sci Signal 3: ra52, 2010.

36. Leone V, D'Angelo D, Ferraro A, et al: A TSH-CREB1-microRNA loop is required for thyroid cell growth. Mol Endocrinol 25: 1819-1830, 2011. 